\title{
HYPOTHESIS ARTICLE
}

\section{PHILOSOPHY OF TIME AND AGING}

\section{Samuel Chiquita}

Summary: This article hypothesis is that the aging process is associated with time. In order to understand the philosophy of aging the concept of time needs to be deeply understood. Firstly, a physical understanding of this process is highlighted, putting in evidence the role of temperature, entropy and speed of motion in time. After, evidence for the association between aging and time is presented.

Keywords: Time, aging, temperature, entropy, speed of motion

\section{INTRODUCTION}

Zeno's paradoxes of motion [1] make us think about what space is and what time is. Motion can be described by speed (v), which is expressed as the ratio between the distance (d) travelled in space by an object in a given time ( $t$ ) interval: $v=d / t$. Heisenberg's uncertainty principle [2] clearly states that speed and position cannot be determined precisely at the same time. This effect becomes evident at the quantum level, putting in evidence Zeno's paradoxes of dividing time and space in infinites. At the macroscopic level Einstein's theory of general relativity [3] expresses the relationship between space and time as a space-time continuum. According to Einstein's twin paradox two twin brothers moving at different speeds will have distinct aging rates [4]. This puts in evidence the importance of space, time and speed of motion in the aging process. Furthermore, with thermodynamic laws is possible to understand the relationship between temperature and entropy in nature. In particular, the third law of thermodynamics clearly states that at absolute zero the entropy of a system tends to zero [5]. Therefore, the stability of a system is dependent on temperature. Bearing this in mind one can think that the aging process might be related with the entropy state of 
an organism since with time living organisms increase their disorganization. The timeasymmetric behavior of macroscopic entities can be assigned to Boltzmann's entropy and is an explanation of the second law of thermodynamics [6]. Life can be maintained over a time period by exchanging entropy with the environment. Nevertheless, as time progresses the entropy of the system reaches a maximum value in which a given organism is said to be death [7]. Since chronological aging is quantified by measuring the time an organism is alive this means that if we can increase the number of years an organism is alive we can influence the aging process. If time is related with space, speed of motion, temperature and entropy we need to be able to influence these variables in order to be able to increase the number of years an organism is alive.

\section{PHILOSOPHY OF TIME AND AGING}

The philosophy of time and aging relies on the following postulates:

\section{Postulate 1}

According to Einstein's theory of relativity time and space form a space-time continuum.

\section{Postulate 2}

In order to fully stop time the following variables shall be considered: temperature, entropy and speed of motion.

\section{Postulate 3}

If time is fully stopped the aging process can be stopped.

The considerations expressed about time are mainly derived from physical laws. Nevertheless, living organisms working principles might not be as straightforward as the physical laws already known. In this case, these considerations provide the basis for a structured reasoning about living matter that needs to be developed. This development will enable to establish mechanisms in which aging can be altered. Nowadays, it has been proved experimentally that time dilation due to motion exists [8]. Moreover, some 
insects show diapause phenotype induced by temperature/photoperiod with an associated increase in lifespan [9]. In humans biological aging can influence the number of years an individual is alive [10]. Several factors can affect human lifespan, including telomere length and lifestyle factors [11]. Therefore, besides the variables already discussed, organisms' age is affected by biological factors such as telomere length, reactive oxygen species, DNA damage, metabolic rate which also need to be taken in consideration in order to increase lifespan. According to the third law of thermodynamics it is possible to reduce the entropy of a system by reducing its temperature. A straightforward application is cryopreservation, which is already applied for preserving human cells and tissues $[12,13]$. This process might help in increasing human lifespan. Nevertheless, the second law of thermodynamics imposes conditions to the entropy value a system can have. This means that as time progresses an isolated macroscopic system evolves towards an increased entropy state. In the limit, we might be able to delay the aging process but we may not be able to stop it.

\section{Philosophy of time and aging}
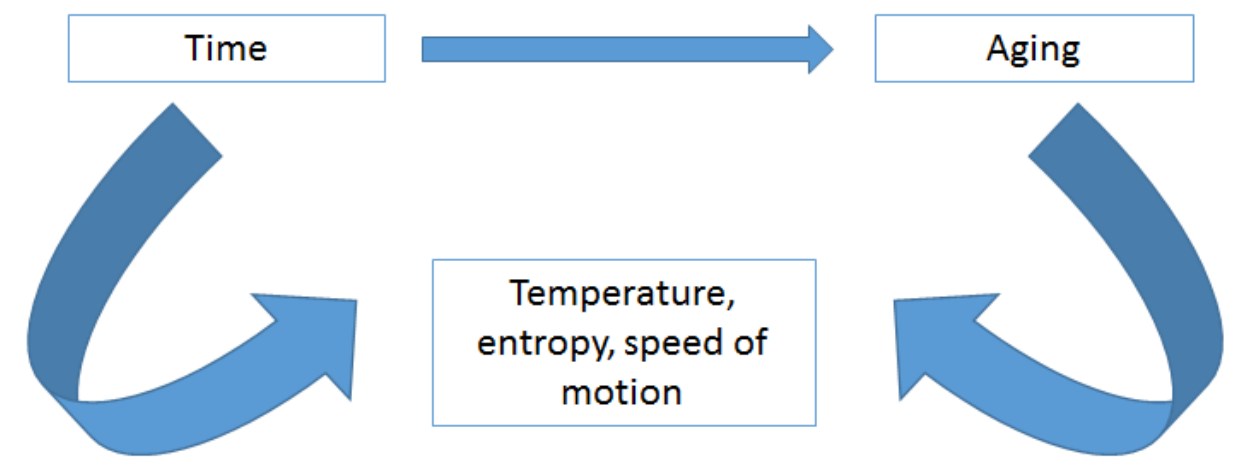

Figure 1 - The aging process is associated with time progression. Moreover, time depends on temperature, entropy and speed of motion. In order to understand the aging process and to delay it all these variables need to be considered. 


\section{REFERENCES}

[1] N. Huggett, "Zeno's paradoxes," 2002.

[2] J. Hilgevoord and J. Uffink, "The uncertainty principle," 2001.

[3] T. A. Ryckman, "Early philosophical interpretations of general relativity," 2001.

[4] P. Pesic, "Einstein and the twin paradox," European journal of physics, vol. 24, no. 6 , p. 585, 2003.

[5] J. Wilks, The third law of thermodynamics, Oxford University Press, 1961.

[6] J. L. Lebowitz, "Boltzmann's entropy and time's arrow," Physics today, vol. 46, no. 9, pp. 32-38, 1993.

[7] E. Schrodinger, "What is life?: the physical aspect of the living cell," 1946.

[8] C.-W. Chou, D. Hume, T. Rosenband and D. Wineland, "Optical clocks and relativity," Science, vol. 329, no. 5999, pp. 1630-1633, 2010.

[9] M. Reis, F. B. Valer, C. P. Vieira and J. Vieira, "Drosophila americana diapausing females show features typical of young flies," PloS one, vol. 10, no. 9, p. e0138758, 2015.

[10] D. W. Belsky, A. Caspi, R. Houts, H. J. Cohen, D. L. Corcoran, A. Danese, H. Harrington, S. Israel, M. E. Levine, J. D. Schaefer and others, "Quantification of biological aging in young adults," Proceedings of the National Academy of Sciences, vol. 112, no. 30, pp. E4104--E4110, 2015.

[11] M. A. Shammas, "Telomeres, lifestyle, cancer, and aging," Current opinion in clinical nutrition and metabolic care, vol. 14, no. 1, p. 28, 2011.

[12] J. Bakhach, "The cryopreservation of composite tissues: principles and recent advancement on cryopreservation of different type of tissues," Organogenesis, vol. 5, no. 3, pp. 119-126, 2009.

[13] J. G. Baust, D. Gao and J. M. Baust, "Cryopreservation: An emerging paradigm change," Organogenesis, vol. 5, no. 3, pp. 90-96, 2009. 